\title{
FS-MAC+: Troca flexível do protocolo de acesso ao meio em redes sem fio empregando aprendizado de máquina
}

\author{
André V. G. S. Gonçalves ${ }^{1}$, Daniel F. Macedo ${ }^{1}$, Luiz F. M. Vieira ${ }^{1}$ \\ ${ }^{1}$ Departamento de Ciência da Computação - Universidade Federal de Minas Gerais (UFMG) \\ Belo Horizonte - MG - Brazil \\ \{andre.gomes, damacedo, lfvieira\}@dcc.ufmg.br
}

\begin{abstract}
Wireless networks operating on open frequencies suffer from external interference and competition for the use of the spectrum, which vary in time and space. In addition, there is a great diversity of applications running on wireless networks, each with different requirements. For example, real-time voice applications require low latency and require low data throughput. On the other hand, file transfer-based applications have higher tolerance to high latency but require higher throughput. Despite this, MAC protocols do not address the dynamism of the environment and applications of today's wireless networks. To reduce this problem, we propose a platform based on machine learning for the online swap of the MAC protocol. This process adapts continuously to variations in the network. The proposal was tested and validated in real wireless environments.
\end{abstract}

Resumo. Em redes sem fio operando em frequências abertas, temos problemas como interferência externa e concorrência pelo uso do espectro, que variam no tempo e no espaço. Além disso, há uma grande diversidade de aplicações rodando sobre redes sem fio, cada qual com diferentes requisitos. Por exemplo, aplicações de voz em tempo real demandam baixa latência e requerem baixa vazão de dados. Por outro lado, aplicações baseadas em transferência de arquivos possuem maior tolerância a alta latência porém requerem maior vazão. Apesar disso, os protocolos MAC não atendem ao dinamismo do ambiente e das aplicações das redes sem fio atuais. Para reduzir esse problema, propomos uma plataforma baseada em aprendizado de máquina para a troca on-line do protocolo MAC. A seleção é contínua, adaptando-se a variações na rede. A proposta foi testada e validada em ambientes reais de redes sem fio.

Palavras-chave: aprendizado de máquina, flexibilização, protocolo MAC, redes sem fio.

\section{Introdução}

Redes sem fio são ambientes dinâmicos e sujeitos a múltiplas fontes de problemas. Interferência, terminais escondidos e variação na qualidade do enlace são alguns exemplos. Esses problemas são agravados ainda mais em redes sem fio operando em frequências abertas, devido à alta concorrência pelo espectro. Adicionalmente, há uma grande diversidade de aplicações rodando em redes sem fio, cada qual com diferentes requisitos. Por exemplo, aplicações de voz em tempo real demandam baixa latência, porém são pouco sensíveis a variações de vazão de dados. Por outro lado, aplicações baseadas em transferência de arquivos possuem menor sensibilidade a variações de latência, porém possuem maior demanda por vazão. 
Protocolos MAC não atendem ao dinamismo do ambiente e diversidade de aplicações nas quais redes sem fio estão submetidas, pois são focados em cenários específicos. Tomemos como exemplo duas classes populares de protocolos MAC: CSMA (Carrier-Sense Multiple Access) e TDMA (Time-Division Multiple Access). Protocolos CSMA são oportunísticos e sem sincronização. Não há alocação de recursos. Protocolos CSMA possuem bom desempenho em cenários de rede sem fio com baixa concorrência e baixa interferência [Sharp et al. 1995]. Por outro lado, protocolos TDMA possuem coordenação e alocação de recursos através da reserva de intervalos de tempo de transmissão para cada nó. Isso beneficia cenários de alta concorrência e alta interferência [Sharp et al. 1995]. Concorrência e interferência são fatores usualmente variáveis em redes sem fio ao longo do tempo e espaço. Portanto, nem protocolos CSMA, nem protocolos TDMA possuem bom desempenho o tempo todo. Outros exemplos de protocolos MAC e seus respectivos cenários específicos de aplicação podem ser encontrados em [Choudhury et al. 2006], [Menouar et al. 2006], [Shengbin and Xiaoliang 2011], [Gopalan and Park 2010] e [Demirkol et al. 2006].

Uma solução para o dinamismo do meio seria um mecanismo de adaptação automático do protocolo MAC de acordo com a condição momentânea da rede sem fio. Apesar de parecer uma proposta distante, atualmente é possível realizar a troca de parâmetros das camadas mais baixas da pilha de protocolos empregando rádios definidos por software (do inglês Software-Defined Radios ou SDR) [Dillinger et al. 2005]. Segundo o Wireless Innovation Forum, mais de $90 \%$ dos equipamentos sem fio comerciais já empregavam em 2011 o conceito de SDR em algum grau [Forum 2011]. Na literatura, vários trabalhos tem sido propostos com SDR: [Sexton et al. 2017], [Bloessl et al. 2015], [Abari et al. 2016], [Buey et al. 2017], [Srisooksai et al. 2017], [Cordeiro et al. 2018] etc.

Nesse contexto, desenvolvemos em trabalhos anteriores o FS-MAC, que é uma plataforma flexível capaz de promover a troca do protocolo MAC em atividade na rede [Cordeiro et al. 2018]. Entretanto, o FS-MAC possui um sistema de seleção do protocolo MAC limitado, baseado em apenas duas métricas de rede, e dependente de parâmetros de configuração fornecidos pelo usuário para ajuste do motor de seleção. O FS-MAC emprega lógica nebulosa para codificação de regras, o que requer um processo laborioso e que demanda conhecimento especializado para a definição de regras efetivas.

A proposta deste artigo, chamada $\mathrm{FS}-\mathrm{MAC}+{ }^{1}$, consiste em um aperfeiçoamento da plataforma FS-MAC. Utilizamos técnicas de aprendizado de máquina para a seleção do protolo MAC mais apropriado. O FS-MAC+ também seleciona o protocolo MAC baseado em um conjunto maior de atributos da rede, permitindo uma decisão mais adaptada ao contexto atual. A seleção do algoritmo de aprendizado de máquina foi feita de forma a reduzir o erro de estimação. Além disso, avaliamos o desempenho do FS-MAC+ em um ambiente real, composto por rádios definidos por software.

As principais contribuições desse trabalho são a criação de uma plataforma inteligente de troca do protoloco MAC que melhor se adapte às condições da rede sem fio, implementação e avaliação da proposta em ambientes de redes sem fio reais, e a disponibilização da implementação de forma gratuita.

Os resultados mostram parecer favorável ao FS-MAC+. Trocas ocorrem de ma-

\footnotetext{
${ }^{1}$ Disponível em https://github.com/andreviniciusgsg/FS-MACplus
} 
neira a beneficiar o melhor desempenho da rede em resposta à alguma alteração em relação à rede sem fio ou ambiente. FS-MAC+ apresenta resultados similares aos resultados do melhor protocolo MAC do momento.

O restante do artigo está organizado da seguinte forma. A seção 2 apresenta os trabalhos relacionados à nossa proposta. A seção 3 descreve a arquitetura do FS-MAC+. Na seção 4 apresentamos a metodologia de avaliação adotada assim como os resultados obtidos. Por último, a seção 5 apresenta as conclusões obtidas assim como propostas de trabalhos futuros.

\section{Trabalhos Relacionados}

Há na literatura alguns trabalhos relacionados à nossa proposta. Listamos aqui os principais trabalhos encontrados. Dedicamos uma subseção ao FS-MAC, que é a base para o desenvolvimento da plataforma FS-MAC+.

[Rhee et al. 2008] propõem a criação de um protocolo MAC híbrido chamado ZMAC que mistura conceitos tanto de TDMA quanto de CSMA. O acesso ao meio é dividido em slots de tempo similarmente ao TDMA, onde cada nó é dono de um slot. Os nós da rede disputam o uso de cada slot de tempo de maneira similar ao CSMA. O nó primeiro escuta ao meio e, caso o meio esteja disponível, transmite. Caso haja disputa entre o dono do slot de tempo e outro nó qualquer, o nó dono tem prioridade em relação ao uso do slot. Como resultado, o Z-MAC alterna implicitamente entre os protocolos TDMA e CSMA. O Z-MAC difere da nossa proposta uma vez que Z-MAC é por si só um protocolo MAC, enquanto o FS-MAC+ é uma plataforma de troca de protocolos MAC. Seria possível, por exemplo, adicionar o protocolo Z-MAC à plataforma FS-MAC+ de modo a selecioná-lo sempre que o cenário for favorável ao seu uso.

AMAC é similar ao FS-MAC+ em termos de possibilitar a troca de protocolos MAC de acordo com o cenário da rede [Huang et al. 2009]. O AMAC alterna o protocolo MAC em uso entre CSMA e TDMA de acordo com a degradação de vazão de dados. A seleção do protocolo MAC é feita sempre que há degradação igual ou superior a $20 \%$ nas últimas cinco medições. Esse método é rudimentar em situações como, por exemplo, a presença de um agente externo gerador de ruído. Nesse caso, ambos os protocolos serão prejudicados pelo ruído, não sendo necessariamente bom executar a troca do protocolo MAC apesar da degradação da vazão. As regras de seleção são fixas e [Huang et al. 2009] não deixa claro quais são os critérios de escolha do próximo protocolo MAC quando a troca é solicitada. O AMAC é limitado em relação ao FS-MAC+, uma vez que a troca do protocolo MAC é baseada apenas em uma métrica de rede (vazão de dados), e não considera métricas importantes da rede, como mencionado anteriormente.

O trabalho de [Qiao et al. 2016] se assemelha à nossa proposta. SMAC utiliza técnicas de aprendizado de máquina supervisionados para seleção do protocolo MAC (DCF ou TDMA). A principal diferença entre SMAC e FS-MAC+ está na metodologia de validação. O trabalho de [Qiao et al. 2016] utiliza simulações computacionais para validação de SMAC, enquanto nosso trabalho é validado experimentalmente em ambientes reais de redes sem fio. Como é sabido, em redes sem fio é muito difícil simular as condições do meio, de forma que os resultados em simulação podem não refletir a realidade de um ambiente de produção. 


\subsection{FS-MAC}

[Cordeiro et al. 2018] é o trabalho base para nossa proposta. FS-MAC consiste em uma plataforma flexível da subcamada MAC. O FS-MAC possui três módulos principais: Sensoriamento, Decisão e Troca. A figura 1 ilustra a arquitetura. O módulo de Sensoriamento é responsável pela coleta de métricas da rede sem fio; o módulo de Decisão é responsável pela seleção do protocolo MAC a ser utilizado; finalmente, o módulo de Troca é responsável pela troca e manutenção do protocolo MAC dentre todos os nós da rede.

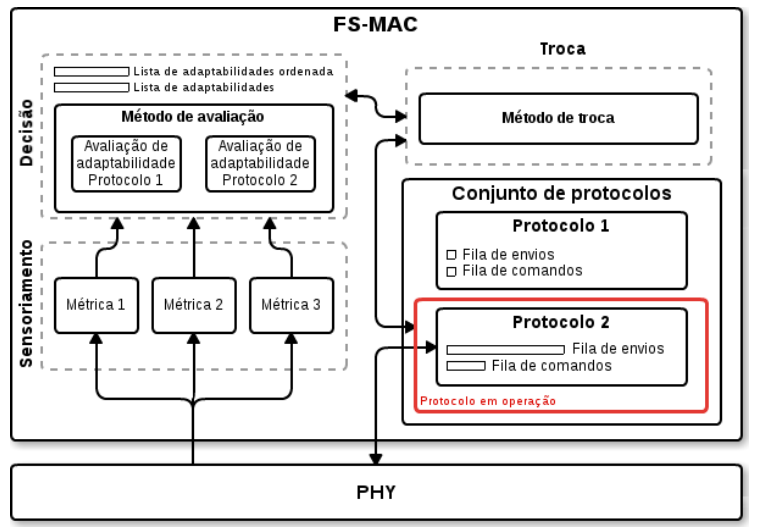

Figura 1. Arquitetura em módulos do FS-MAC [Cordeiro et al. 2018].

O módulo de Decisão proposto por [Cordeiro et al. 2018] é baseado em lógica fuzzy e limitado. É necessário a configuração manual de regras de inferência para seleção do protocolo MAC. Essas regras devem ser definidas considerando-se tanto o cenário da rede sem fio quanto características de cada protocolo MAC. Adicionalmente, o módulo de Decisão do FS-MAC considera apenas duas métricas de rede, número de nós transmissores e latência. Portanto, FS-MAC necessita de um agente externo para definição das regras de inferência e possui apenas duas métricas para a tomada de decisão.

O FS-MAC+ proposto neste tarbalho difere-se de FS-MAC em relação aos módulos de Sensoriamento e Decisão. Nossa proposta implementa um número maior de métricas. Assim, temos uma visão mais fiel às condições da rede sem fio. Segundo, apresentamos um módulo de Decisão mais sofisticado, com a utilização de métodos de aprendizado de máquina, não sendo necessário um agente externo para análise e definição de parâmetros de decisão.

\subsection{Comparação entre os métodos propostos na literatura}

A tabela 1 resume as principais características dos trabalhos relacionados encontrados na literatura. Ressaltamos aqui os principais diferenciais da nossa proposta em relação aos demais trabalhos. FS-MAC+ é o único a fornecer flexibilidade de seleção. Isso quer dizer que o critério de seleção pode ser alterado de acordo com as necessidades do momento. Por exemplo, a demanda por alta vazão de dados pode ser alterada para minimização da latência dependendo da natureza das aplicações rodando na rede. A partir desse momento, FS-MAC+ deixará de selecionar o protocolo MAC de maior vazão e passará a selecionar o procolo MAC que fornece a menor latência.

Apenas FS-MAC+ e SMAC possuem um sistema sofisticado de seleção do protocolo MAC baseado em aprendizado de máquina com múltiplas métricas de entrada. 
Tabela 1. Comparação entre FS-MAC+ e literatura

\begin{tabular}{|l|c|c|c|c|c|}
\hline & Z-MAC & AMAC & SMAC & FS-MAC & FS-MAC+ \\
\hline Protocolos & CSMA, TDMA & CSMA, TDMA & DCF, TDMA & CSMA, TDMA & CSMA, TDMA \\
\hline Extensibilidade MAC & Sim & Sim & - & Sim & Sim \\
\hline Flexibilidade de seleção & - & - & - & Sim \\
\hline Seleção & - & $\begin{array}{c}\text { Degradação } \\
\text { de vazão }\end{array}$ & $\begin{array}{c}\text { Aprendizado } \\
\text { de máquina }\end{array}$ & $\begin{array}{c}\text { Lógica fuzzy } \\
\text { de máquina }\end{array}$ \\
\hline Regras de seleção & - & $\begin{array}{c}\text { Limiar pré- } \\
\text { estabelecido }\end{array}$ & Classificação & $\begin{array}{c}\text { Regras de } \\
\text { inferência }\end{array}$ & Regressão \\
\hline Quantidade de métricas & - & 1 & 12 & 2 & 5 \\
\hline Validação & Experimental & Experimental & Simulação & Experimental & Experimental \\
\hline Implementação & TinyOS & Gnu Radio & OPNET & Gnu Radio & Gnu Radio \\
\hline
\end{tabular}

SMAC utiliza um modelo de classificação baseado em 12 métricas de rede. Vale ressaltar que o grande número de métricas de entrada é mascarado pela alta correlação entre algumas dessas métricas. Por exemplo, quatro dessas métricas correspondem à métrica de vazão de dados agregadas de diferentes formas [Qiao et al. 2016]. FS-MAC+ utiliza modelos de regressão baseados em 5 métricas distintas. Além disso, SMAC é validado através de simulações computacionais enquanto FS-MAC+ é validado experimentalmente em ambientes de redes sem fio reais. Simulações computacionais dificilmente simulam todas as condições do meio, podendo não condizer com a realidade.

Por último, apenas FS-MAC e FS-MAC+ disponibilizam as implementações e exemplos práticos dos respectivos trabalhos. Isso facilita a análise e a criação de futuras propostas de aperfeiçoamento mesmo por não-autores.

\section{FS-MAC+}

FS-MAC+ é uma plataforma inteligente de troca de protocolo MAC baseada em técnicas de aprendizado de máquina. A plataforma é uma extensão da plataforma FS-MAC, cuja arquitetura é ilustrada pela figura 1. Nossa proposta é focada nos módulos de Sensoriamento e de Decisão. O processo de seleção do protocolo MAC feito pelo módulo de Decisão é centralizado e só é executado por um nó, denominado nó coordenador [Cordeiro et al. 2018]. É também responsabilidade do nó coordenador manter apenas um protocolo MAC operando em toda a rede.

As principais vantagens da plataforma FS-MAC+ em relação ao FS-MAC e aos demais trabalhos relacionados são a utilização de métricas de rede distintas, o motor de seleção do protocolo MAC baseado em técnicas de aprendizado de máquina e a validação da proposta em ambientes reais de rede sem fio. Abaixo discutiremos em mais detalhes cada um desses tópicos.

\subsection{Módulo de Sensoriamento}

O módulo de Sensoriamento é responsável pela coleta de métricas de rede a serem utilizadas pelo módulo de Decisão. Assim como em FS-MAC, há dois tipos de métricas. O primeiro consiste em métricas centralizadas, que são coletadas exclusivamente pelo nó coordenador. Um exemplo de métrica centralizada é o número de nós na rede. O segundo são métricas distribuídas, geradas por todos os nós da rede e enviadas ao nó coordenador. Latência e vazão de dados são exemplos de métricas distribuídas.

FS-MAC+ utiliza cinco métricas, cada qual com o objetivo de representar uma característica da rede. A lista de métricas utilizadas pelo FS-MAC+ é apresentada abaixo. 
1. Vazão de dados: A vazão de dados representa a quantidade de informação trafegando pela rede;

2. Número de nós: Quantidade de nós pertencentes e ativos na rede;

3. Latência: Tempo necessário para enviar um pacote com sucesso ao nó de destino.

4. SNR (Signal Noise Ratio): SNR representa a qualidade do canal de comunicação e é dado pela razão entre o nível de sinal recebido e o nível de ruído local.

5. RNP (Required Number of Packet retransmissions): Quantidade média de retransmissões por pacote [da Silva 2016]. Um alto valor de RNP indica problemas na entrega de pacotes, seja por interferência (interna ou externa) ou mal funcionamento do nó;

Número de nós é a única métrica centralizada em FS-MAC+. Todas as demais são calculadas localmente por todos os nós e, posteriormente, enviadas ao nó coordenador. Métricas distribuídas tem a vantagem de representar melhor as condições da rede como um todo. Por exemplo, o cálculo centralizado de SNR talvez não represente a qualidade do sinal em todos os nós. É possível que alguns nós da rede estejam em uma zona de alta interferência, enquanto o nó coordenador esteja em uma região com baixa interferência. Nesse caso, é provável que a seleção do protocolo MAC não beneficie os nós com baixo SNR, prejudicando-os ou até mesmo os impedindo de transmitir informação. Métricas distribuídas atenuam esse tipo de problema ao levar em consideração os valores reportados por cada nó.

A taxa de amostragem das métricas do módulo de Sensoriamento é de cinco segundos. Essas amostras podem ser agregadas de diferentes formas no nó coordenador, sendo possível: máximo, mínimo, soma, média simples e média móvel. A agregação é importante para as métricas pois o desempenho e a condição da rede podem variar drasticamente. Assim, o método de decisão requer uma suavização dos dados de entrada para que a sua precisão seja maior. A granularidade da agregação pode ser alterada para cada métrica, e a configuração padrão é agregação média simples com granularidade de 30 segundos.

\subsection{Módulo de Decisão}

O módulo de Decisão é responsável pela seleção do protocolo MAC a ser utilizado na rede de acordo com os valores das métricas reportadas pelo módulo de Sensoriamento. A seleção é feita através de técnicas de aprendizado de máquina supervisionado. Utilizamos modelos de regressão para estimar o desempenho de cada protocolo MAC dado uma métrica alvo. A métrica alvo pode ser estabelecida de acordo com as necessidades da aplicação rodando sobre a rede. Por exemplo, se a rede tem como foco principal transmitir dados de voz em tempo real, é provável que a métrica alvo seja latência. Nesse caso, o objetivo seria obter o protoloco MAC de menor latência. Outra possível métrica alvo é a vazão de dados. Aplicações baseadas em transferência de arquivos são tolerantes à variações de latência mas possuem alta demanda por vazão. Assim, o objetivo seria obter o protocolo MAC que resultasse na maior vazão de dados.

A modelagem do problema empregando um preditor de desempenho de cada protocolo, ao invés de um preditor que indica qual será o protocolo indicado, é empregada para permitir a extensibilidade da proposta. Assim, ao invés de ser necessário retreinar todo o módulo de decisão a cada novo protocolo adicionado, faz-se necessário somente 
prover um modelo de regressão de desempenho para o novo protocolo. De forma similar, o modelo de decisão pode ser trocado a qualquer momento, por exemplo de um modelo que prioriza a vazão para um modelo que priorize a latência.

A troca do protocolo MAC é feita sempre que a predição do protocolo atual for inferior à predição de outro protocolo dado um fator de tolerância. $\mathrm{O}$ fator de tolerância tem como objetivo evitar o efeito ping-pong, passível de ocorrer em situações quando ambos os protocolos possuem desempenho similar. Por padrão, adotamos um fator de tolerância de $20 \%$ obtido empiricamente.

\subsection{Módulo de Troca}

O módulo de Troca do FS-MAC+ funciona de maneira similar ao módulo de Troca proposto por [Cordeiro et al. 2018]. A única diferença consiste na substituição do mecanismo de adaptabilidade do módulo de Troca do FS-MAC pelo fator de tolerância do módulo de Decisão do FS-MAC+. No mais, o módulo de Troca é igual. O módulo de Troca do nó coordenador difunde mensagens de controle periodicamente, informando o protocolo MAC em uso. Nos demais nós, o módulo de Troca chaveia entre protocolos MAC de acordo com as mensagens de controle recebidas. As mensagens de controle geram overhead de aproximadamente $2 \%$ como apontado por [Cordeiro et al. 2018].

\subsection{Implementação}

A implementação da plataforma FS-MAC+ é feita em rádios definidos por software da família USRP Ettus através da plataforma de processamento digital de sinais GNU Radio. Mais informações sobre rádios definidos por software e GNU Radio podem ser encontradas em [da Silva et al. 2015]. Assim como FS-MAC, FS-MAC+ utiliza a implementação do protocolo ZigBee de [Bloessl et al. 2013] como camada física em relação à pilha de protocolos de rede. O módulo de Sensoriamento foi programado em linguagem $\mathrm{C}++$, enquanto o módulo de Decisão foi programado em linguagem Python.

A implementação em rádios definidos por software possibilita a validação da plataforma em ambientes reais.

\subsection{Escolha dos algoritmos de aprendizado de máquina}

Os algoritmos de aprendizado de máquina utilizados são descritos em [Pedregosa et al. 2011]. Há oito modelos de regressão implementados no módulo de Decisão, sendo eles: Linear, Linear por SVR (Support Vector Regression), SVR, NuSVR, KNN (K-Nearest Neighbors regression), DT (Decision Tree), NNet (Neural Network regression) e GBRT (Gradient Boosted Regression Trees). Também há disponível os protocolos MAC CSMA/CA e TDMA puros. Por último, o módulo de Decisão do FS-MAC+ também conta com o motor de seleção baseado em lógica fuzzy do FS-MAC [Cordeiro et al. 2018].

Avaliamos o desempenho dos algoritmos de aprendizado de máquina considerando um conjunto de dados coletados durante experimentos. Trinta porcento desses dados são aleatoriamente reservados para o conjunto de validação cruzada. A figura 2 ilustra os valores percentuais médios de RMSE (do inglês Root Mean Square Error) para cada um dos modelos implementados no módulo de Decisão. Trata-se da razão entre RMSE e 
o valor médio da medição. Observe que os algoritmos DT (Decision Tree) e NNet (Neural Network regression) apresentam menor desvio percentual entre o valor do preditor e o valor real para o conjunto de validação cruzada. Por apresentarem menores erros, DT e NNet são os algoritmos avaliados nesse trabalho.

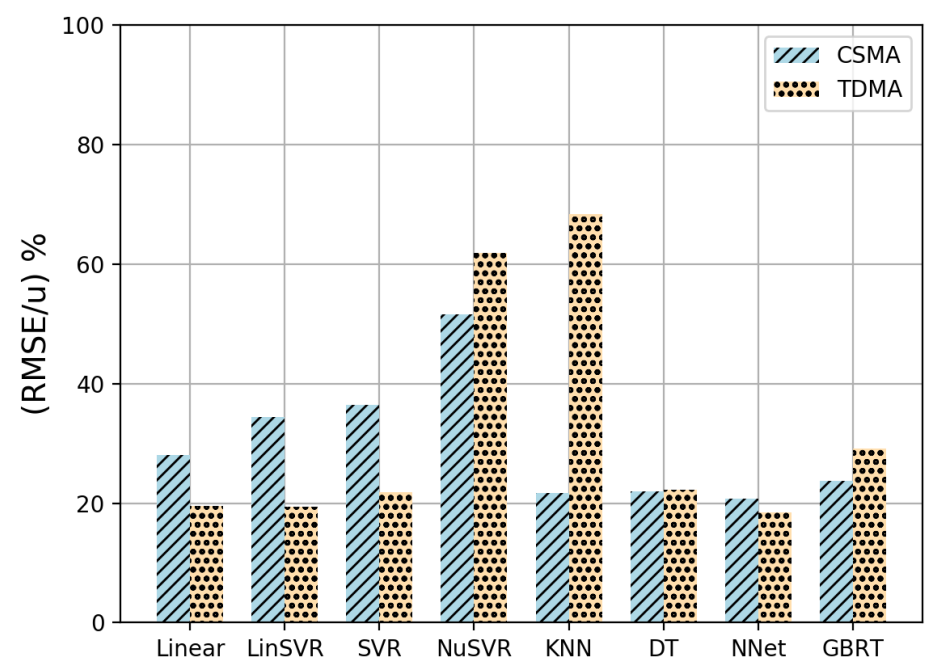

Figura 2. Avaliação dos algoritmos de aprendizado de máquina. Gráfico $(R M S E / \mu)$ por modelo de predição.

\section{Avaliação}

Avaliamos o desempenho do FS-MAC+ em cenários reais de rede sem fio. Propomos três cenários distintos de avaliação para validação da proposta. Primeiro, avaliamos o desempenho do FS-MAC+ em relação ao nível de concorrência. Para isso, adicionamos incrementalmente novos nós na rede. Segundo, analisamos um cenário com a presença de ruído. Por último, avaliamos a plataforma para uma métrica alvo diferente.

Os dados de treinamento utilizados foram coletados em distintos cenários de rede. O objetivo é cobrir o maior número possível de possibilidades comportamentais da rede. Cada medição consiste em uma tupla dos seguintes valores: id do protocolo MAC, vazão de dados, número de nós, latência, RNP e SNR. Uma medição corresponde a amostras agregadas como mostra a tabela 2. A escolha dessa configuração foi feita empiricamente com o objetivo de evitar variações bruscas e momentâneas que possam ocorrer na rede. Por exemplo, é possível que algum nó deixe de enviar amostras em um dado momento, seja porque não obteve acesso ao meio para transmissão ou por falhas na rede. A agregação do número de nós visa atenuar esse problema, contabilizando sempre todos os nós que reportaram qualquer tipo de dado dentro da janela de 30 segundos. No total, foram geradas 416 medições para o processo de treinamento, que correspondem a aproximadamente $3: 30 \mathrm{~h}$ de conhecimento da rede, considerando-se a granularidade de 30 segundos.

Tanto a avaliação quanto o processo de treinamento foram feitos utilizando-se rádios definidos por software da família USRP Ettus modelos B200 e B210. Utilizamos 
Tabela 2. Agregações usadas na avaliação.

\begin{tabular}{|l|c|c|}
\hline & Agregação & Granularidade (seg) \\
\hline Vazão de dados & Média por nó & 30 \\
\hline Número de nós & Máximo & 30 \\
\hline Latência & Média por nó & 30 \\
\hline RNP & Média por nó & 30 \\
\hline SNR & Média por nó & 30 \\
\hline
\end{tabular}

as 8 USRPs disponíveis no testbed FUTEBOL UFMG da Universidade Federal de Minas Gerais $^{2}$. A figura 3 mostra uma USRP Ettus (caixa azul) do testbed FUTEBOL UFMG, uma das USRPs utilizadas nesse trabalho.

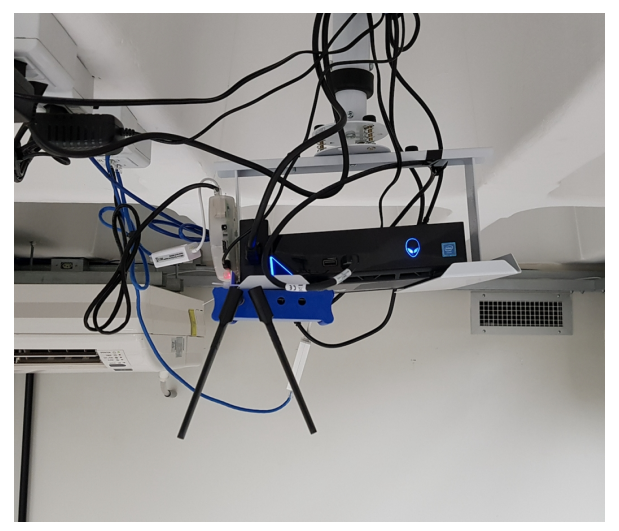

Figura 3. Exemplo de nó sem fio com USRP Ettus disponível no testbed FUTEBOL UFMG.

A avaliação do FS-MAC+ é feita através do contraste entre os resultados obtidos com FS-MAC+ (algorítimos DT e NNet) e os resultados obtidos com CSMA/CA puro, TDMA puro e FS-MAC. Os resultados são médias aritméticas simples com os respectivos desvios padrões. A metodologia dos testes é descrita em cada cenário nas próximas seções.

\subsection{Cenário 1: Desempenho vs. Número de nós}

Nesse cenário avaliamos o desempenho do FS-MAC+ em relação ao número de nós presentes na rede. O objetivo é maximizar a vazão de dados. A topologia adotada é similar à ideia de gateway padrão, onde toda a comunicação é geralmente feita entre nós e gateway. O nó coordenador representa o nó gateway. Todos os demais nós transmitem informação exclusivamente para o nó coordenador. A figura 4 representa a disposição dos nós, onde o nó 0 é o coordenador e os demais nós são inseridos sucessivamente em ordem crescente de $i d$.

A figura 5 mostra os resultados obtidos para esse primeiro cenário. Um novo nó é inserido na rede a cada 200 segundos. Os resultados são médias com os respectivos desvios padrões das medições obtidas a cada 30 segundos dentro da janela de 200 segundos entre a inserção de novos nós. O eixo vertical representa a vazão total na rede, dada em quadros por segundo. O gráfico mostra um melhor desempenho do protocolo MAC

\footnotetext{
${ }^{2}$ Mais informações em http: / / futebol.dcc.ufmg.br/
} 


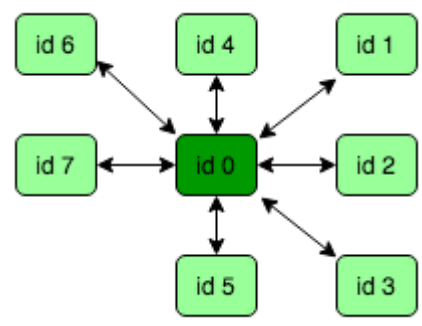

Figura 4. Disposição dos nós para avaliação.

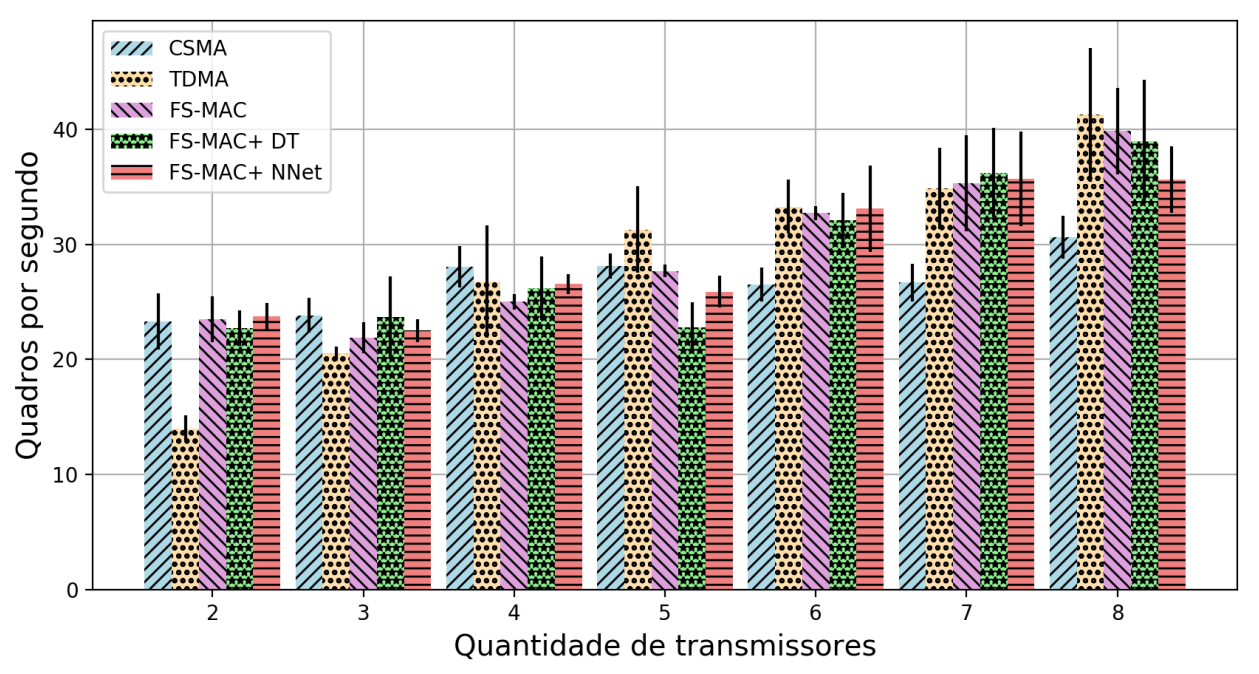

Figura 5. Vazão (quadros por segundo) vs. Número de nós.

CSMA/CA quando o número de nós é inferior a 4. Quando o número de nós é maior que 4, o desempenho do TDMA é superior. Esse resultado é compatível com o reportado por [Sharp et al. 1995], sendo CSMA melhor em ambientes de baixa concorrência e TDMA melhor em cenários de maior concorrência. Observe que tanto FS-MAC quanto FS-MAC+ tendem a acompanhar o desempenho do melhor protocolo. Há uma maior variação dos resultados quando o número de nós é igual a 5. Nesse caso, FS-MAC+ apresenta desempenho consideravelmente menor que ambos CSMA/CA e TDMA. Essa variação está relacionada ao processo de troca do protocolo MAC. Quando a troca ocorre, há uma pequena perda da desempenho devido a overheads e momentânea desestabilização durante o período de transição. Além disso, FS-MAC+ DT é penalizado por uma troca extra de protocolos MAC quando o número de nós está entre 4 e 5 . Essa perda momentânea de desempenho é compensada pelo melhor desempenho subsequente, similar ao desempenho do protocolo TDMA.

\subsection{Cenário 2: Adição de ruído}

A adição de ruído prejudica a qualidade do canal de comunicação entre nós. Nos referimos a ruído de maneira generalizada como qualquer tipo de sinal que atrapalhe a comunicação dentro da rede. Há várias causas: ruído ambiente, ruído térmico, sinais de outras redes, sinais gerados por equipamentos eletrônicos na mesma faixa de frequência e até mesmo algum nó defeituoso operando fora dos padrões estabelecidos. 
Para avaliar esse ambiente, modificamos um nó para operar sem qualquer tipo de controle de acesso ao meio. Esse nó defeituoso foi posicionado entre dois outros nós comunicando entre si. Em relação à figura 4, o nó 0 é o coordenador, o nó 1 comunica normalmente e o nó 2 faz o papel do nó defeituoso.

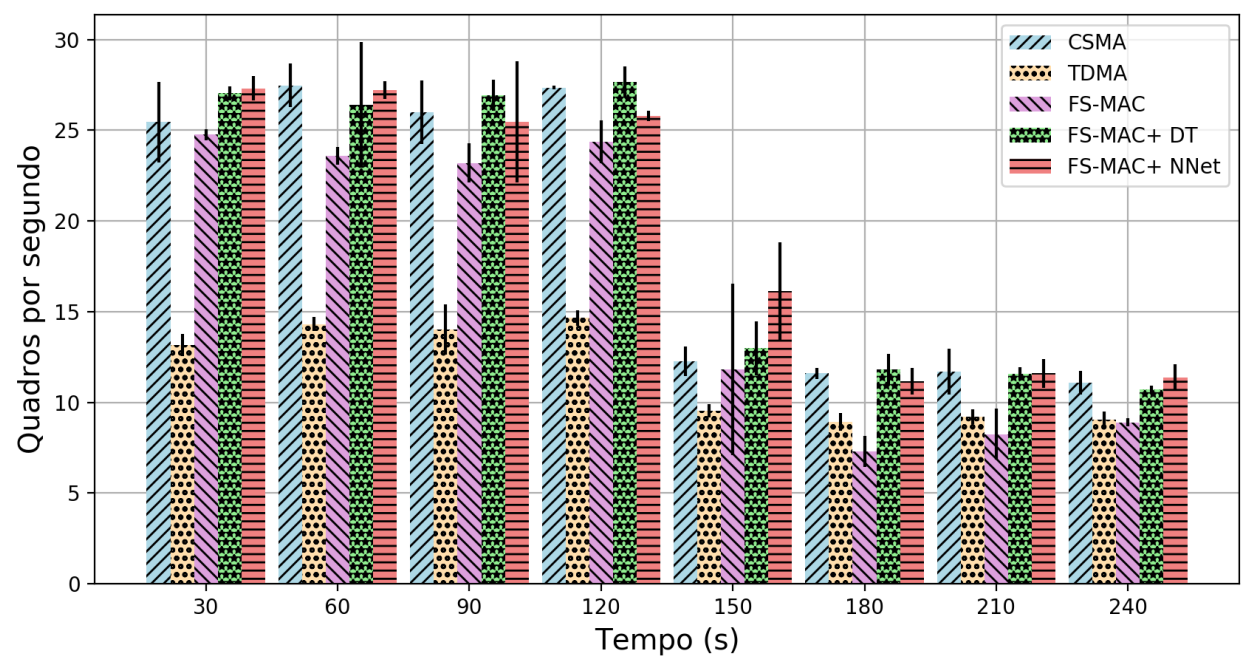

Figura 6. Vazão (quadros por segundo) vs. Tempo (segundos).

A figura 6 mostra os resultados para esse segundo cenário. O nó defeituoso é inserido na rede entre os segundos 120 e 150 . O processo foi repetido três vezes e os resultados são as médias do total de vazão de cada repetição com o respectivo desvio padrão. Tanto o protocolo CSMA/CA quanto o TDMA são prejudicados pela presença do nó defeituoso, sendo o CSMA/CA o maior prejudicado com maior queda de vazão. Contudo, o protocolo CSMA/CA ainda é significativamente melhor que o TDMA. Resultados mostram que FS-MAC+ opera de acordo com o melhor protocolo em ambos algoritmos avaliados (DT e NNet). Há uma variação considerável de comportamento no instante 150. Observe que FS-MAC+ NNet possui desempenho consideravelmente superior à ambos CSMA e TDMA. Isso provavelmente ocorre devido à variações bruscas durante a inserção do nó defeituoso, gerando um comportamento anormal tanto para FS-MAC+ NNet quanto para os demais. Por outro lado, FS-MAC passa a operar conforme o protocolo TDMA após a inserção do nó defeituoso, resultando em menor vazão.

\subsection{Cenário 3: Diferente métrica alvo}

Vazão de dados pode não ser a métrica alvo apropriada para todas as situações. Em algumas situações, possuir uma rede com latência mínima é mais desejável que manter uma rede de alta vazão. Em alguns outros casos, uma rede confiável com baixas taxas de retransmissão traz maiores benefícios. Nessa seção avaliamos esse último cenário.

Uma rede com baixas taxas de retransmissão é útil em situações onde recursos energéticos são escassos. Considere o cenário de uma rede alimentada por baterias e painéis solares. Considere também períodos nos quais a incidência de luz solar é baixa. É de extrema importância poupar bateria e evitar que os nós sejam desligados por falta de energia. Transmissões demandam energia e, portanto, não é desejável que transmissões 
mal-sucedidas sejam realizadas. Consideramos esse cenário na perspectiva de minimizar a métrica RNP, razão entre o número de retransmissões pelo número de transmissões bem sucedidas [da Silva 2016].

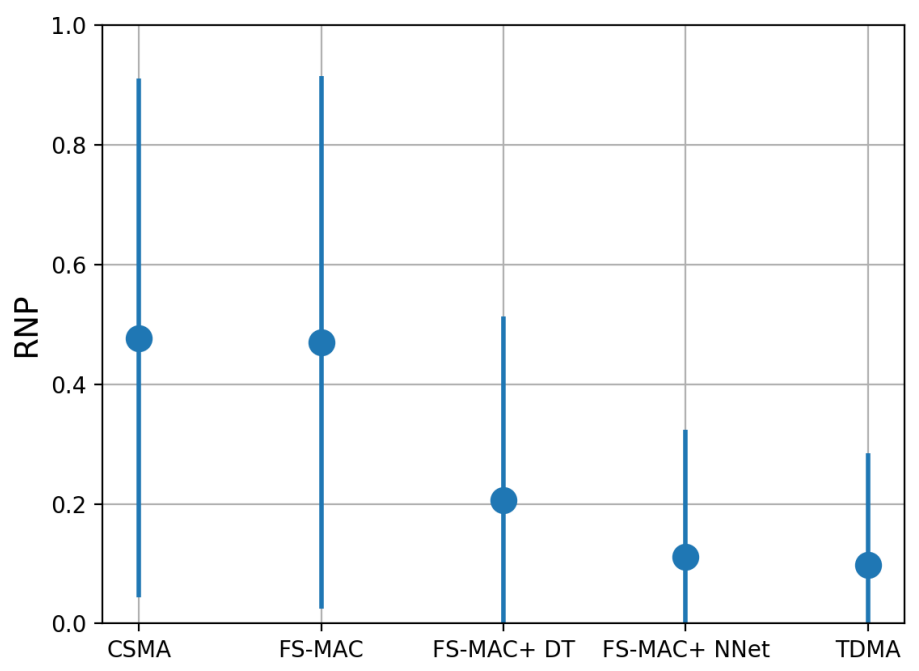

Figura 7. RNP por protoloco.

A figura 7 mostra os resultados comparando CSMA/CA, FS-MAC, FS-MAC+ (DT e NNet) e TDMA. Para cada um, coletamos o valor médio de RNP durante um intervalo de 5 minutos e repetimos o processo 4 vezes. O gráfico da figura 7 representa a média das 4 repetições com o respectivo desvio padrão. Trata-se da comunicação entre dois nós. O valor médio de FS-MAC é bem similar ao resultado do CSMA/CA puro. Ambos apresentam taxas mais altas de retransmissão e desperdiçariam bateria no contexto energético. FS-MAC+ DT possui valores intermediários devido ao chaveamento entre CSMA/CA e TDMA em alguns períodos. Ainda assim é válido ressaltar que o valor médio está mais próximo do TDMA que do CSMA, sendo FS-MAC+ DT ainda razoavelmente eficiente. Por último, FS-MAC+ NNet e TDMA apresentam maior eficiência uma vez que possuem os menores valores de retransmissão e, portanto, poupam energia ao evitarem transmissões fracassadas.

A alteração da métrica alvo pode ser generalizada para outras métricas. Assumimos comportamentos similares com a utilização de métricas alvos tais como latência ou SNR, ou ainda mesmo outras possíveis métricas passíveis de serem implementadas.

\section{Conclusões e trabalhos futuros}

Neste artigo propomos uma plataforma de troca flexível do protocolo de acesso ao meio em redes sem fio. Nossa proposta, FS-MAC+, utiliza técnicas de aprendizado de máquina supervisionado para seleção do melhor protocolo MAC de acordo com as condições da rede. Avaliamos dois dos oitos algoritmos de aprendizado de máquina implementados, sendo os dois selecionados (DT e NNet) os algoritmos que apresentaram menor erro de predição. Resultados mostram que FS-MAC+ é capaz de selecionar o melhor protocolo MAC em distintas situações. FS-MAC+ também apresenta melhores resultados em relação ao FS-MAC, plataforma base do nosso trabalho. 
FS-MAC+ foi avaliado em três cenários distintos. No primeiro, FS-MAC+ apresentou resultados similares ao FS-MAC. Ambos foram capazes de selecionar o melhor protocolo MAC, protocolo de maior vazão de dados, em relação ao nível de concorrência na rede. No segundo cenário, o FS-MAC+ apresentou melhores resultados, sempre selecionando o protocolo MAC de maior vazão. Por último, avaliamos um cenário de minimização da métrica RNP. O FS-MAC+ apresentou os menores valores de RNP, sendo que o FS-MAC+ NNet obteve resultados equivalente aos obtidos com TDMA puro, protocolo MAC com menor RNP. Portanto, a avaliação ressalta a capacidade de adaptação do FS-MAC+ ao cenário da rede sem fio, através da seleção do melhor protocolo MAC.

Por fim, registramos aqui algumas propostas de trabalhos futuros. FS-MAC+ utiliza técnicas de aprendizado de máquina supervisionado. Essa abordagem pode ser ineficiente em cenários com pouca correlação com o conjunto de treinamento. Pretendemos incorporar aprendizado semi-supervisionado ao FS-MAC+, tornando-o mais robusto e capaz de melhorar o desempenho com o tempo. Segundo, a plataforma FS-MAC+ foi testada em cenários de rede sem fio operando de acordo com o padrão ZigBee. Pretentemos avaliá-la também em cenários compatíveis com o padrão IEEE 802.11. Além disso, pretendemos investigar cenários abordando mobilidade, múltiplos saltos e maior densidade de nós.

\section{Agradecimentos}

Gostaríamos de agradecer o suporte das seguintes agências brasileiras de fomento: CAPES, CNPq e FAPEMIG. Além disso, agradecemos também ao projeto FUTEBOL (Projeto europeu H2020, no. 688941).

\section{Referências}

Abari, O., Hassanieh, H., Rodreguiz, M., and Katabi, D. (2016). Poster: A millimeter wave software defined radio platform with phased arrays. In Proceedings of the $22 \mathrm{Nd}$ Annual International Conference on Mobile Computing and Networking, MobiCom '16, pages 419-420, New York, NY, USA. ACM.

Bloessl, B., Leitner, C., Dressler, F., , and Sommer, C. (2013). A GNU radio-based IEEE 802.15.4 testbed. http://www.ccs-labs.org/bib/bloessl2013gnu/ bloessl2013gnu.pdf. Acessado:20-12-2017.

Bloessl, B., Sommer, C., and Dressler, F. (2015). Power matters: Automatic gain control for a software defined radio IEEE $802.11 \mathrm{a} / \mathrm{g} / \mathrm{p}$ receiver. In 2015 IEEE Conference on Computer Communications Workshops (INFOCOM WKSHPS), pages 25-26.

Buey, C., Magounaki, T., Ferrero, F., Ratajczak, P., Lizzi, L., and Kaltenberger, F. (2017). MIMO antenna performance assessment based on open source software defined radio. In 2017 11th European Conference on Antennas and Propagation (EUCAP), pages 644-647.

Choudhury, R. R., Yang, X., Ramanathan, R., and Vaidya, N. H. (2006). On designing MAC protocols for wireless networks using directional antennas. IEEE Transactions on Mobile Computing, 5(5):477-491.

Cordeiro, J. R. S., Lanza, E., and D. F. Macedo, L. F. M. V. (2018). FS-MAC: A flexible MAC platform for wireless networks. In IEEE Wireless Communications and Networking Conference. 
da Silva, W. S. (2016). Técnicas de estimação de qualidade de enlace para comunicação máquina a máquina.

da Silva, W. S., Cordeiro, J. R. S., Macedo, D. F., Vieira, M. A. M., Vieira, L. F. M., and Nogueira, J. M. S. (2015). Introdução a rádios definidos por software com aplicações em GNU radio. Minicurso apresentado no XXXIII Simpósio Brasileiro de Redes de Computadores e Sistemas Distribuídos.

Demirkol, I., Ersoy, C., and Alagoz, F. (2006). MAC protocols for wireless sensor networks: a survey. IEEE Communications Magazine, 44(4):115-121.

Dillinger, M., Madani, K., and Alonistioti, N. (2005). Software defined radio: Architectures, systems and functions. John Wiley \& Sons.

Forum, W. I. (2011). Software defined radio - rate of adoption. http://www . wirelessinnovation.org/sdr_rate_of_adoption.

Gopalan, S. A. and Park, J.-T. (2010). Energy-efficient MAC protocols for wireless body area networks: Survey. In International Congress on Ultra Modern Telecommunications and Control Systems, pages 739-744.

Huang, K. C., Jing, X., and Raychaudhuri, D. (2009). MAC protocol adaptation in cognitive radio networks: An experimental study. In 2009 Proceedings of 18th International Conference on Computer Communications and Networks, pages 1-6.

Menouar, H., Filali, F., and Lenardi, M. (2006). A survey and qualitative analysis of mac protocols for vehicular ad hoc networks. IEEE Wireless Communications, 13(5):3035 .

Pedregosa, F., Varoquaux, G., Gramfort, A., Michel, V., Thirion, B., Grisel, O., Blondel, M., Prettenhofer, P., Weiss, R., Dubourg, V., Vanderplas, J., Passos, A., Cournapeau, D., Brucher, M., Perrot, M., and Duchesnay, E. (2011). Scikit-learn: Machine learning in python. J. Mach. Learn. Res., 12:2825-2830.

Qiao, M., Zhao, H., Wang, S., and Wei, J. (2016). MAC protocol selection based on machine learning in cognitive radio networks. In 2016 19th International Symposium on Wireless Personal Multimedia Communications (WPMC), pages 453-458.

Rhee, I., Warrier, A., Aia, M., Min, J., and Sichitiu, M. L. (2008). Z-MAC: A hybrid MAC for wireless sensor networks. IEEE/ACM Transactions on Networking, 16(3):511-524.

Sexton, C., Kaminski, N. J., Marquez-Barja, J. M., Marchetti, N., and DaSilva, L. A. (2017). 5G: Adaptable networks enabled by versatile radio access technologies. IEEE Communications Surveys Tutorials, 19(2):688-720.

Sharp, B. A., Grindrod, E. A., and Camm, D. A. (1995). Hybrid tdma/csma protocol for self managing packet radio networks. In Universal Personal Communications. 1995. Record., 1995 Fourth IEEE International Conference on, pages 929-933.

Shengbin, L. and Xiaoliang, Z. (2011). A Survey on MAC Layer in Ad Hoc Wireless Networks, pages 691-699. Springer Berlin Heidelberg, Berlin, Heidelberg.

Srisooksai, T., i. Takada, J., and Saito, K. (2017). Portable wide-band channel sounder based software defined radio for studying the radio propagation in an outdoor environment. In 2017 International Symposium on Antennas and Propagation (ISAP), pages $1-2$. 